\title{
Classification of Normal, Ictal and Inter-ictal EEG via Direct Quadrature and Random Forest Tree
}

\author{
Enas Abdulhay $^{1} \cdot$ Maha Alafeef $^{1} \cdot$ Arwa Abdelhay $^{2} \cdot$ Areen Al-Bashir $^{1}$
}

Received: 27 June 2016/Accepted: 4 November 2016/Published online: 19 June 2017

(C) Taiwanese Society of Biomedical Engineering 2017

\begin{abstract}
This paper presents an accurate nonlinear classification method that can help physicians diagnose seizure in electroencephalographic (EEG) signal characterized by a disturbance in temporal and spectral content. This is accomplished by applying four steps. First, different EEG signals containing healthy, ictal and seizure-free (inter-ictal) activities are decomposed by empirical mode decomposition method. The instantaneous amplitudes and frequencies of resulted bands (intrinsic mode functions, IMF) are then tracked by the direct quadrature method (DQ). In contrast to other approaches, DQ cancels the effect of amplitude modulation on frequency calculation. The dissociation between instantaneous amplitude and frequency information is therefore fully achieved to avoid features confusion. Afterwards, the Shannon entropy values of both sets of instantaneous values (amplitudes and frequencies)—related to every IMF-are calculated. Finally, the obtained entropy values are classified by random forest tree. The proposed procedure yields $100 \%$ accuracy for (healthy)/(ictal) and $98.3-99.7 \%$ for (healthy)/ (ictal)/(interictal) classification problems. The suggested method is hence robust, accurate, fast, user-friendly, data driven with open access interpretability.
\end{abstract}

Enas Abdulhay

ewabdulhay@just.edu.jo

1 Department of Biomedical Engineering, Faculty of Engineering, Jordan University of Science and Technology, P.O.Box 3030, Irbid 22110, Jordan

2 Department of Water and Environmental Engineering, Faculty of Natural Resources Engineering, German Jordanian University, P.O.Box 35247, Amman 11180, Jordan
Keywords EEG $\cdot$ Forest tree $\cdot$ Ictal $\cdot$ Direct quadrature . Decomposition $\cdot$ Entropy $\cdot$ Instantaneous

\section{Introduction}

Electroencephalography (EEG) is a medical technique that reads scalp electrical activity generated by brain structures. It is an accurate tool for identification of various types of abnormalities in brain. Epilepsy seizure is one of those complex abnormalities detected by EEG. It is a disturbed electrochemical release in a large cell population. That affects the quality of life of the patient, causing social impairment and a higher risk of death [1]. The spectral and temporal content analysis of EEG signals provides helpful information about the nature of seizure. However, the tool of analysis applied to EEG must be adapted to the characteristics of non-stationary non-linear brain activities.

Several approaches have been applied to EEG signals with the intention of identification of normal, ictal and inter-ictal activities; the latter of which being seizure-free segments occurring between seizure (ictal) fragments. The studies utilize time-domain, frequency-domain, time-frequency and spatial features [2,3]. In [4], a difference in the synchronization level has been observed between EEG seizure and seizure-free intervals. The seizure EEG signals were found to be less random, more nonlinear-dependent, more stationary with comparatively different amplitude level [5-9]. Consequently, every tool utilised for seizure pattern recognition should have the capability to take these main variations into account. In [10], empirical mode decomposition (EMD) [11] has been employed to extract the inherent sub-bands (intrinsic mode functions, IMF) of a number of EEG signals. The mean frequencies of the resulted IMFs are then computed from the Fourier-Bessel 
series and used as the main features of classification. In the works of [1, 12-21], EMD has also been used as the first step. Nonetheless, the exploited features are different. The authors exploited a variety of parameters related to the obtained IMFs: the mean frequencies calculated by Hilbert Transform, the local patterns, the variation coefficients, the fluctuation indices, the entropy measures, the area calculated from the analytic signals, difference plots and phase space representations. In [22], EEG sub-bands have been realized by filtering. Approximate entropy values are subsequently calculated. In [23-26], Wavelet Transform (WT) has been the chosen method for sub-bands calculation. In [27-29], entropy values of EEG components, found by WT, were selected as classification features. In [30], fractional energy on specific Short-Fourier Transform windows has been the feature used for seizure classification. In [31], approximate entropy of EEG amplitude values has been calculated and classified using neural networks. The authors of [32] exploited the properties of Fast Fourier Transform to classify epileptiform EEG using decision tree classifier. In [33], fractional energy values of EEG components, found by pseudo-Wigner-Ville distribution, have been classified via neural networks. In [34], Lyapunov exponents of EEG signals have been utilized as features serving for seizure pattern recognition. The last step in all of the previous studies is the statistical processing of extracted features in order to attempt seizure pattern recognition. Various methods have been applied: nearest neighbour classifiers, decision trees, neural networks, support vector machines, and adaptive neuro-fuzzy systems [33].

The processing of seizure segments necessitates a tool that can adapt to EEG non-stationary and non-linear characteristics and does not imply pre-models $[10,13,14]$. Fourier Transform assumes stationary characteristics. Short-Fourier (SFT) and Wavelet Transforms (WT) assume linear properties as indicated by the first author of the present work in [35]. Furthermore, involving a specific mother wavelet in EEG processing implies a pre-model for analysis and spectral/time resolution. Investigations based on SFT and WT should therefore be complex enough in order to compensate the points of weakness and to turn into data-driven. On the other hand, nonlinear empirical mode decomposition can adaptively and intuitively represent non-stationary signals as sums of zero-mean locally symmetric mono-components AM-FM components (IMFs) [11]. The extracted components are speculatively associated with specific physiological aspects of the phenomenon investigated. EMD method is data driven, intuitive, not time consuming, does not need a predefined model and does not involve concepts of frequency or time resolution.

As indicated, a number of studies have exploited the advantages of EMD for EEG seizure analysis. The highest obtained accuracy values were found when both local temporal and spectral features were utilized [12]. However, the effect of temporal amplitude modulation on extracted spectral features leaded to a reduction of accuracy. In the present paper, direct quadrature (DQ) method is applied to EEG IMFs in order to extract instantaneous amplitudes and frequencies features. In contrast to other approaches, DQ cancels the effect of amplitude modulation on frequency calculation. The dissociation between instantaneous amplitude and frequency information is therefore fully achieved to avoid features confusion. Shannon entropy values of resulted instantaneous values are subsequently calculated.

Open-interpretability and fast processing are essential characteristics that should be included by every EEG seizure analyzer. The highest classification accuracy values have been obtained in previous works $[25,33]$ when neural networks were exploited. However, the inherent classifier pathways in neural networks are relatively "Black boxes" with slow algorithms. Forest random tree is therefore utilized in the present work for features classification. In contrast to neural networks, transparency of tree classifier is important advantage that can help physicians understand the underlying mechanisms in seizure. Furthermore, fast treatment is a promising benefit for eventual seizure prediction.

\section{Materials and Methods}

The overall procedure of the present work is illustrated in Fig. 1. EEG signals (normal, ictal and inter-ictal) are decomposed by EMD. Resulted IMF are then analyzed by direct quadrature method in order to calculate instantaneous amplitudes and frequencies. Shannon entropy of issued instantaneous values are then classified by random forest tree. Finally, classification results are statistically assessed. In the following sections, details about used EEG dataset, decomposition, direct quadrature elements, entropy calculation, feature classification and statistical assessments, respectively, are presented.

\subsection{Dataset}

The EEG dataset presented in [36] is used. The data set includes single channel EEG from healthy and epileptic subjects. The data has five subsets denoted as A_Z, B_O, C_N, D_F and E_S, each containing 100 single channel recordings, each one having $23.6 \mathrm{~s}$ in duration. The sampling frequency of the data is $173.61 \mathrm{~Hz}$. The subsets A_Z and B_O have been recorded extra-cranially. They have been acquired using surface EEG recordings of five healthy volunteers with eyes open and closed respectively. Subsets 


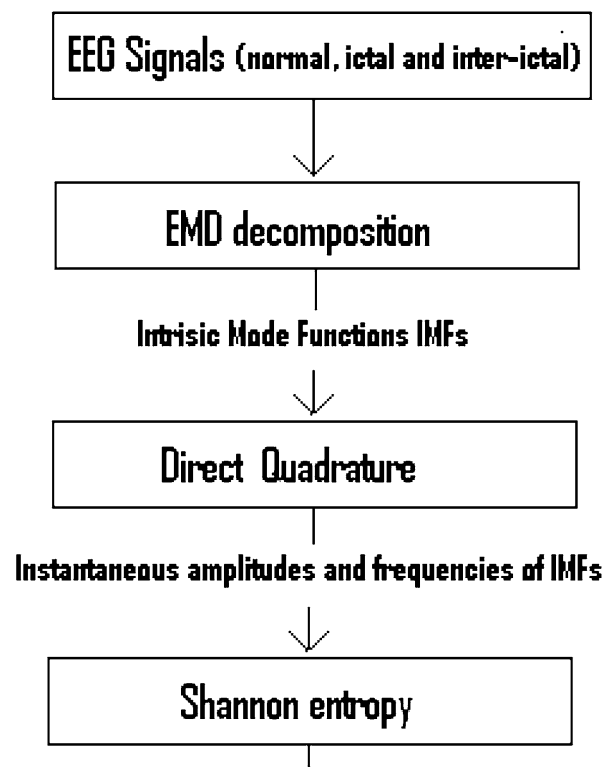

entropy values of instantaneous amplitudes and frequencies of lMFs

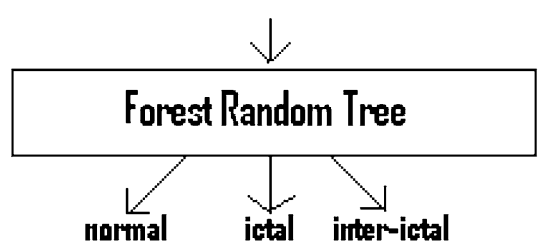

Fig. 1 Suggested steps of EEG signals classification

D_F and C_N have been measured in seizure-free intervals from five patients in epileptic zone and from hyppocampal formation of opposite hemisphere of the brain, respectively. The subset E_S contains seizure activity selected from all recording sites exhibiting ictal activity [12]. The data bandwidth is [0.5-85] Hz. In the present paper, the subsets A_Z, C_N, D_F and E_S are used.

\subsection{Decomposition}

EMD, developed in [11], is a method applied to extract all the oscillatory modes (IMF) embedded in a signal in nonstationary or non-linear conditions. It is data driven, has no difficulties associated with resolution issues and its extracted modes are related to inherent processes. In every extracted IMF, the maximum allowed difference between the number of extrema and the number of zero crossings is one. Besides, the local average of the upper and lower envelopes is zero. These properties permit subsequent calculation of instantaneous frequency and amplitude. The sifting process for extracting IMFs from a signal consists of: first, the identification of all of the maxima and minima. Second, the generation of upper and lower envelopes by cubic spline interpolation and the calculation of point-bypoint mean from the envelopes. Third, the extraction of the detail which is the result of subtraction of the obtained point-by-point mean from the signal. The detail should satisfy the two previously mentioned IMF properties in order to be considered as an IMF. Fourth, the replacement of the original signal with the residual (signal-detail); it is to be considered as the signal for the subsequent IMF calculation. However, if the detail does not meet the requirements, the steps 1-3 should be repeated (iterated) and applied to the detail until it satisfies the two criteria. Finally, the original signal can be expressed as the summation of all of the resulted amplitude modulated-frequency modulated (AM-FM) details and the final residual.

In the present paper, decomposition of used EEG signals has been realized by MATLAB. The maximum number of sifting iterations is 2000 .

\subsection{Direct Quadrature}

The DQ [37] principle is based on the separation of the two effects of amplitude and frequency modulation. Normalization helps remove the effect of amplitude modulation in order to measure the correct instantaneous frequency by the Hilbert transform. Amplitude normalization is performed relative to the envelope of the IMF considered as given by the following equation:

$f_{1}(k)=\frac{I M F(k)}{e_{1}(k)} ; f_{2}(k)=\frac{f_{1}(k)}{e_{2}(k)} \cdots f_{n}(k)=\frac{f_{n-1}(k)}{e_{n}(k)}$

where $n$ is the number of normalizations performed. The term IMF $(k)$ represents the kth sample of IMF. $f_{n}(k)$ is the frequency modulated component. $e_{n}(k)$ is the envelope passing through the maxima of absolute values of $f_{\mathrm{n}-1}(k)$ and conducted by the same approach used to calculate the envelope in EMD. The selected number of successive normalizations $n$ in the present work is 5. Hilbert transform can then be calculated in order to find the correct instantaneous frequency and amplitude [12]. The proposed method is applied by MATLAB to all IMFs resulted from every EEG signal decomposition. Instantaneous frequency and amplitude values can be found by Hilbert transform $[38,39]$ as the instantaneous pulsation and amplitude of the complex analytic signal. The imaginary part of the analytic signal can be calculated by the following formula:

$y(t)=\frac{1}{\pi} \lim _{\in \rightarrow 0^{+}}\left(\int_{t-1 / \epsilon}^{t-\epsilon} \frac{x(\tau)}{t-\tau} d \tau+\int_{t+\epsilon}^{t+1 / \epsilon} \frac{x(\tau)}{t-\tau} d \tau\right)$

where $\mathrm{x}(\mathrm{t})$ is the resulted IMF and is considered as the real part.

\subsection{Shannon Entropy}

Shannon entropy is given by: 
$H=-\sum_{i} p_{i} \log _{b} p_{i}$

where $p i$ is the probability of a value $i$ showing up in a stream of data. Shannon entropy values of the resulted instantaneous frequencies and amplitudes, of the IMFs issued from all used EEG signals, have been calculated by MATLAB.

\subsection{Classification and Validation}

The classification has been realized by Forest Random tree [40] and $k$-fold stratified cross-validation approaches via WEKA (Waikato Environment for Knowledge Analysis) software. A random tree considers randomly a number of chosen attributes at each node. In $k$-fold cross-validation, the original sample is randomly partitioned into $k$ equal sized subsamples. Of the $k$ subsamples, a single subsample is retained as the validation data for testing the model, and the remaining $k-1$ subsamples are used as training data. The cross-validation process is then repeated $k$ times (the folds), with each of the $k$ subsamples used exactly once as the validation data. The $k$ results from the folds can then be averaged (or otherwise combined) to produce a single estimation. In stratified k-fold cross-validation, the folds are selected so that the mean response value is approximately equal in all the folds. The main reason for using cross-validation instead of using the conventional validation (e.g. partitioning the data set into two sets of $70 \%$ for training and $30 \%$ for test) is that the root mean square error on the training set in the conventional validation is not a useful estimator of model performance and thus the error on the test data set does not properly represent the assessment of model performance [41]. Crossvalidation combines (averages) measures of fit (prediction error) to correct for the optimistic nature of training error and derive a more accurate estimate of model prediction performance [42]. Cross validation yields a confusion matrix that indicates true positive, true negative, false positive, false negative rates for every class. The following three types of pattern recognition have been carried out:

\subsubsection{Healthy/ictal Recognition}

A number of 200 signals from A_Z and E_S (100 signals from every dataset) have been used. As every signal has been decomposed into $N$ IMFs, the total number of IMFs is $200 * N$. Every IMF has its related calculated instantaneous frequencies and amplitudes for which entropy values are calculated. The features matrix has therefore a dimension of $(200 * 2 * N)$. The matrix entries have been classified into two classes: normal and ictal. Classification is achieved by Forest Random tree with 40 unlimited depth trees and tenfold cross-validation.

\subsubsection{Healthy/Ictal/Inter-ictal (F) Recognition}

A number of 300 signals from A_Z, E_S and D_F (100 signals from every dataset) have been used. As every signal has been decomposed into $N$ IMFs, the total number of IMFs is $300 * N$. The features matrix has therefore a dimension of $(300 * 2 * N)$. The sub-matrices related to numbers of investigated IMFs scales ranging from $l$ to $N-1$ have also been examined. Each sub-matrix has been classified into three classes: normal, ictal and inter-ictal (epileptic zone). Classification is achieved by Forest Random tree and tenfold cross-validation with 30 unlimited depth trees.

\subsubsection{Healthy/Ictal/Inter-ictal (F), Inter-ictal (N) Recognition}

A number of 400 signals from A_Z and E_S, D_F and C_N have been used. As every signal has been decomposed into $N$ IMFs, the total number of IMFs is $400 * N$. The features matrix has therefore a dimension of $(400 * 2 * N)$. The matrix entries have been classified into three classes: normal, ictal and inter-ictal (epileptic zone) with inter-ictal (opposite hemisphere). Classification is achieved by Forest Random tree with 30 unlimited depth trees and 20-fold cross-validation.

\subsection{Attribute Selection}

The contribution weight of features is studied by WEKA to find the most significant features. CFS supervised attribute subset evaluator (selector) has been used with simple genetic search. Crossover probability, number of generations and mutation probability values are $0.6,20$ and 0.033 , respectively. Attribute selector evaluates the worth of a subset of attributes by considering the individual predictive ability of each feature along with the degree of redundancy between them. Subsets of features that are highly correlated with the class while having low inter-correlation are preferred. It identifies locally predictive attributes and iteratively adds attributes with the highest correlation with the class as long as there is not already an attribute in the subset that has a higher correlation with the attribute in question. It treats missing as a separate value. The attribute selection mode is a tenfold stratified cross validation.

\subsection{Classification Assessment}

Statistical assessment has been conducted by MedCalc based on resulted confusion matrix-issued from cross validation-in order to evaluate the obtained classification results. The calculated statistical descriptors are: accuracy, sensitivity, specificity, positive likelihood ratio, negative 

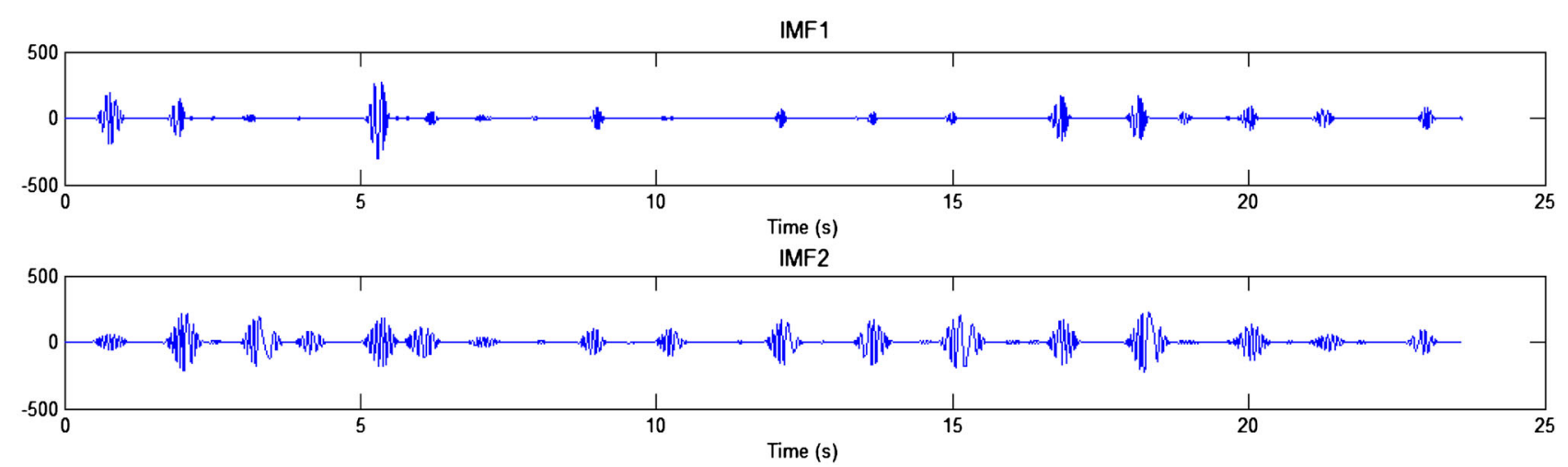

IMF3
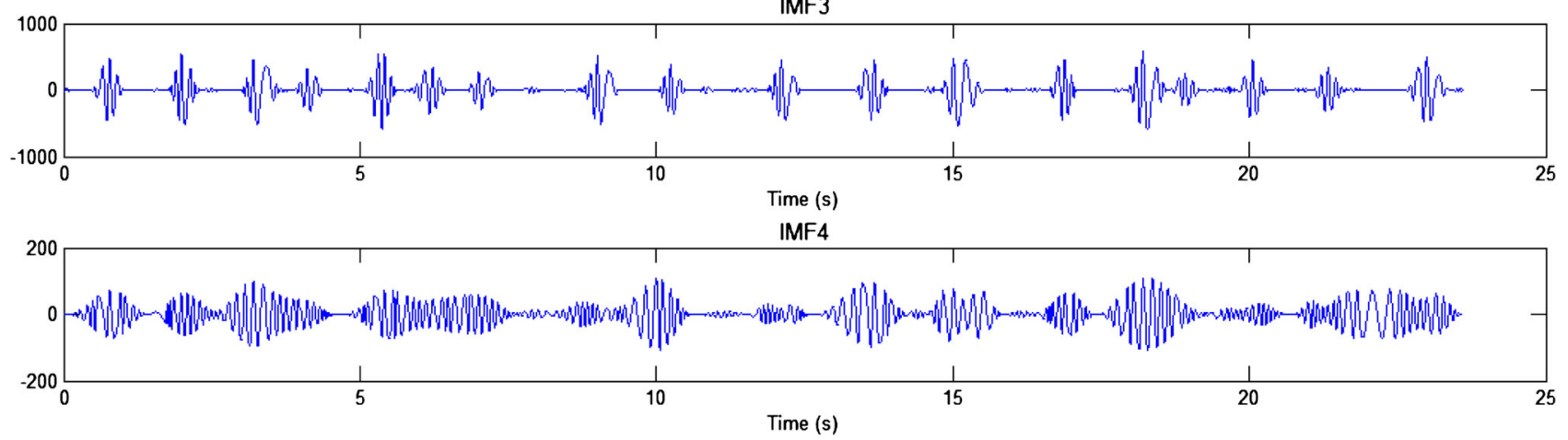

IMF5

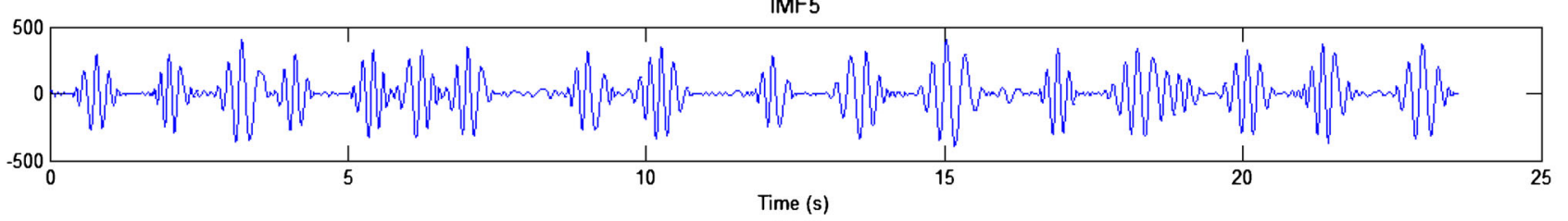

IMF6
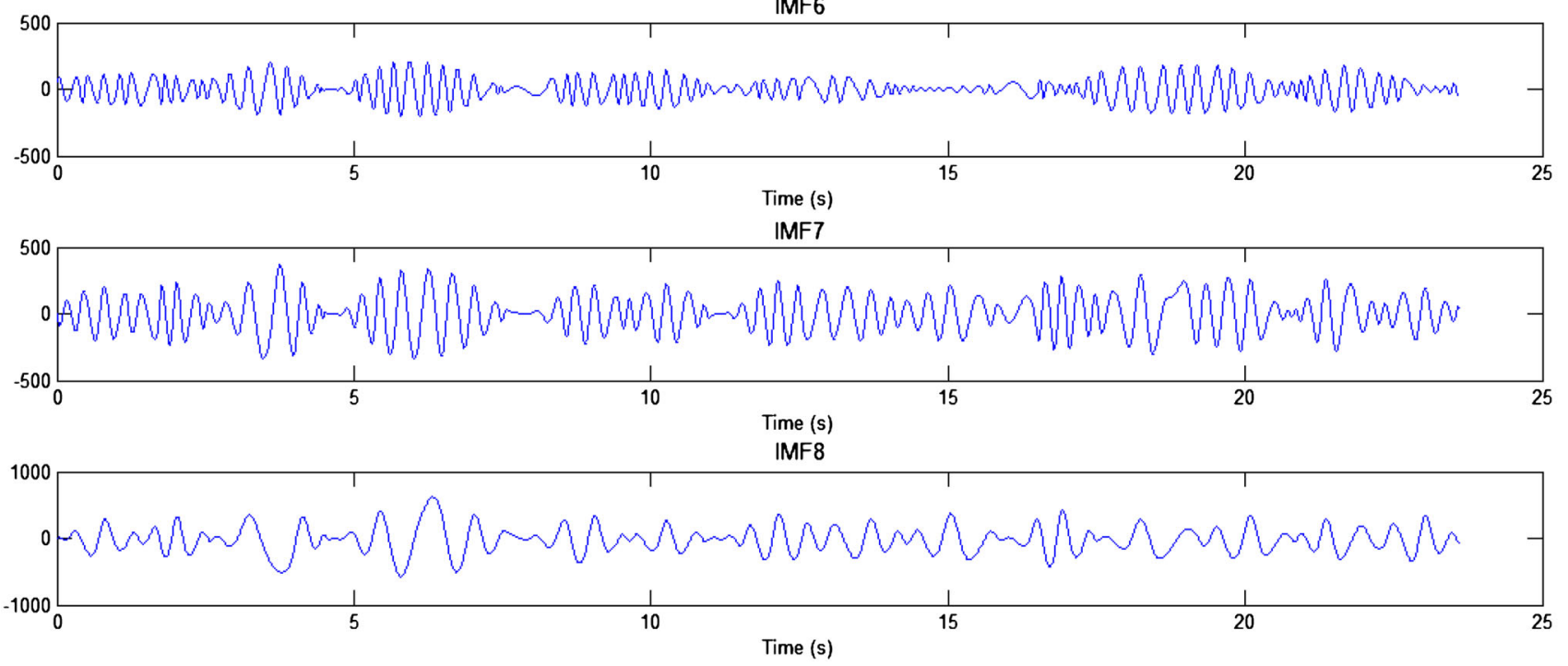

Fig. 2 IMFs issued from EMD decomposition of a signal from the inter-ictal dataset F

likelihood ratio, class prevalence, positive predictive value and negative predictive value using the formula of the Bayes' theorem as follows below, where $a$ : true positive, $b$ : false negative, $c$ : false positive and $d$ : true negative. In the case of our studied classification problem, the unit of a, b, c and d is 'EEG signal'. For example, if ' $a$ ' is 5 , it means that 5 EEG signals are correctly classified in a specific class. 

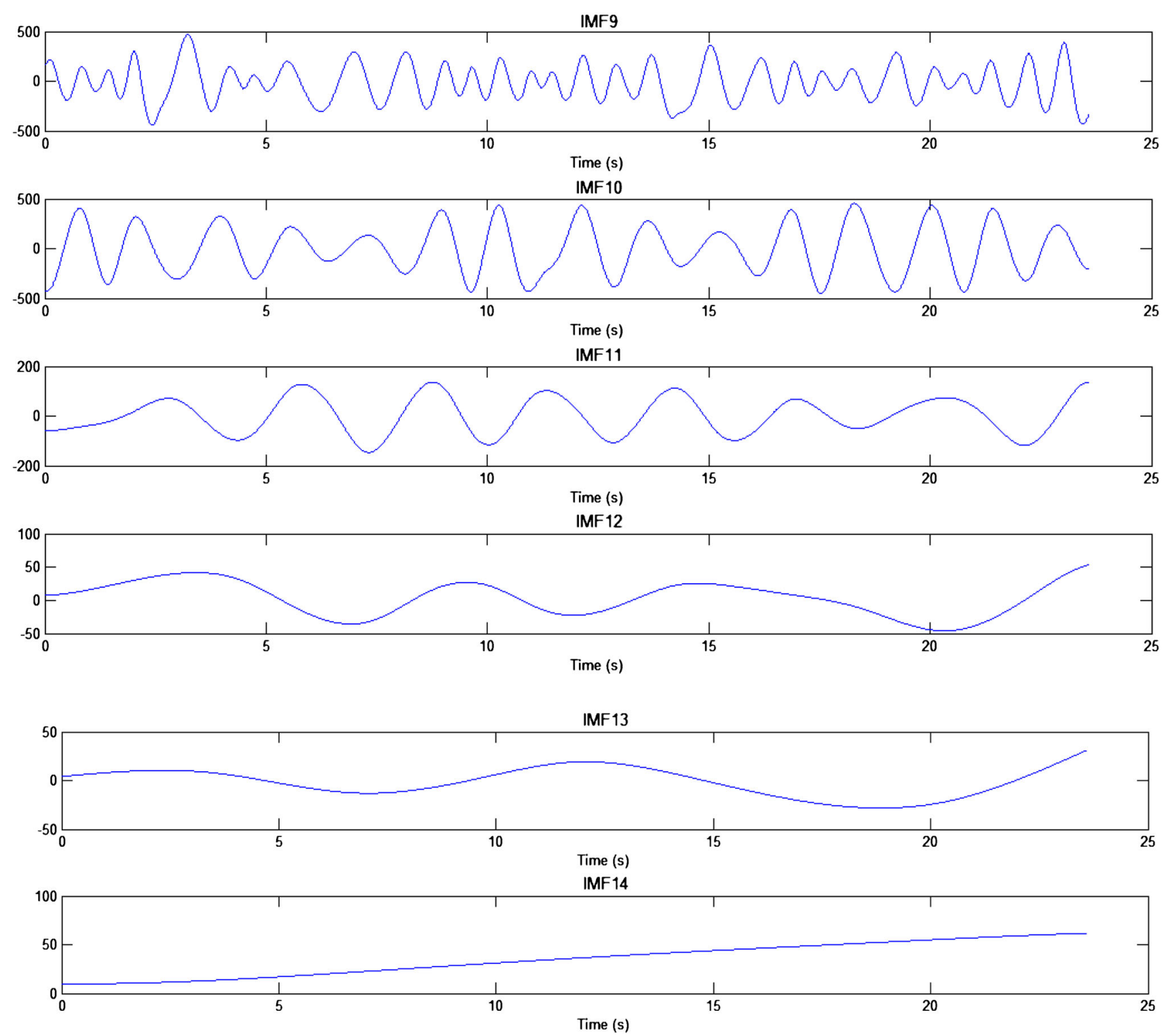

Fig. 2 continued

Sensitivity: $\frac{a}{a+b}$

Specificity: $\frac{d}{c+d}$

Positive Likelihood Ratio: $\frac{\text { Sensitivity }}{100-\text { Specificity }}$

The PLR value is undefined when specificity is $100 \%$. In this case, the cell related to positive likelihood ratio value (in the tables of results section) will not be filled.

Negative likelihood ratio: $\frac{100-\text { Sensitivity }}{\text { Specificity }}$
(4) Class prevalence: $\frac{a+b}{a+b+c+d}$

(5) Positive predictive value: $\frac{a}{a+c}$

Negative predictive value: $\frac{d}{b+d}$

The above values are dimensionless. However, in the results section, they will be presented after multiplication by $100 \%$.

The statistical evaluation of the classification is achieved for every class. Evaluation for a certain class is 
Table 1 A sample of entropy values of instantaneous frequencies and amplitudes for the different IMFs scales and classes

\begin{tabular}{|c|c|c|c|c|c|c|c|c|c|c|c|c|c|c|}
\hline $\mathrm{f} 1$ & f2 & f3 & $\mathrm{f} 4$ & \multicolumn{2}{|l|}{ f5 } & \multicolumn{2}{|l|}{ f6 } & \multicolumn{2}{|l|}{ f7 } & \multicolumn{2}{|l|}{ f8 } & \multicolumn{2}{|l|}{ f9 } & $\mathrm{f} 10$ \\
\hline 26.234 & -14.3255 & -8.18907 & -5.11555 & \multicolumn{2}{|c|}{-3.15719} & \multicolumn{2}{|c|}{-1.81752} & \multicolumn{2}{|c|}{-1.12649} & \multicolumn{2}{|c|}{-0.33245} & \multicolumn{2}{|c|}{-0.30425} & -0.14302 \\
\hline-23.6085 & -14.6468 & -9.34704 & -4.75152 & \multicolumn{2}{|c|}{-2.7267} & \multicolumn{2}{|c|}{-1.25881} & \multicolumn{2}{|c|}{-0.4931} & \multicolumn{2}{|c|}{-0.26888} & \multicolumn{2}{|c|}{-0.18206} & -0.1572 \\
\hline-22.8174 & -18.7521 & -14.9188 & -14.1352 & \multicolumn{2}{|c|}{-11.0446} & \multicolumn{2}{|c|}{-10.5738} & \multicolumn{2}{|c|}{-5.96037} & \multicolumn{2}{|c|}{-3.99148} & \multicolumn{2}{|c|}{-2.25113} & -1.2081 \\
\hline-20.4639 & -18.2399 & -15.9141 & -10.4648 & \multicolumn{2}{|c|}{-6.53297} & \multicolumn{2}{|c|}{-3.61999} & \multicolumn{2}{|c|}{-1.45707} & \multicolumn{2}{|c|}{-0.97} & -0 & 51422 & -0.30243 \\
\hline-25.3151 & -15.5234 & -9.29302 & -4.96115 & -2.314 & & -1.21 & & -0 & 082 & -0 . & 6471 & -0 & 2023 & -0.04874 \\
\hline-24.9437 & -15.5585 & -7.24111 & -3.84451 & -1.793 & & -1.04 & & -0 & 207 & -0 & 4181 & -0 & 1288 & -0.09514 \\
\hline-22.5967 & -21.9553 & -17.1007 & -11.0622 & -8.560 & & -5.17 & & -4 & 393 & -2 & 3222 & -1 & 33832 & -0.34815 \\
\hline-23.6338 & -17.9279 & -15.3715 & -14.3116 & $-12.82^{\prime}$ & & -8.51 & & -6 & & -5 & 9912 & & 10582 & -1.74943 \\
\hline a1 & $\mathrm{a} 2$ & a3 & $a$ & a5 & a6 & & a7 & & a8 & & a9 & & a10 & $\mathrm{Cla}$ \\
\hline 1368 & 7 & 5 & 8.75 & 30 & & 2 & & & & & 5444 & & 126.258 & $\mathrm{Z}$ \\
\hline 2526.03 & 5 & 84564 & 30453.76 & 51823.66 & & 69 & 28 & 7.14 & 40 & & 972.6 & & 394.9333 & $\mathrm{Z}$ \\
\hline 2229.34 & 10743 & 123461.3 & 141203.6 & 1463 & & & 23 & 3 & 270 & & 236765 . & & 146016.8 & $\mathrm{~S}$ \\
\hline 897 & 1 & 27 & 77.7 & 16 & & .7 & 5 & 50.1 & 3. & & 73 & & 52238.02 & $S$ \\
\hline 28510.76 & 59321.6 & 101214.8 & 97729.42 & 111044.7 & & 44 & 41 & +3 & 87 & & 4192.90 & & -398.189 & $\mathrm{~N}$ \\
\hline 17755.41 & 57205.45 & 105249.3 & 99832.31 & 125892.3 & & .92 & 457 & 17 & 221 & & 17725.2 & & 2014.66 & $\mathrm{~N}$ \\
\hline 138158.4 & 304258.2 & 1166573 & 149820.7 & 945386.2 & 33 & 27.7 & 546 & 34.7 & 103 & & 676868. & & 683494.8 & F \\
\hline 110005 & 53915.71 & 95477.99 & 124394.5 & 428052.5 & 17 & 370.3 & 219 & 41.8 & $4705^{\circ}$ & & 411193. & & 737564.9 & F \\
\hline
\end{tabular}

$Z$ healthy, $S$ ictal, $F$ inter-ictal (seizure zone), $N$ inter-ictal (opposite hemisphere), $f, a$ entropy of instantaneous frequency and amplitude

Table 2 Results of classification (normal/ictal EEG)

\begin{tabular}{lll}
\hline Statistic (overall accuracy $=100 \%)$ & Class: normal & Class: ictal \\
\hline Sensitivity & $100 \%$ & $100 \%$ \\
Specificity & $100 \%$ & $100 \%$ \\
Positive likelihood ratio & & \\
Negative likelihood ratio & 0 & 0 \\
Class prevalence & $50.00 \%$ & $50.00 \%$ \\
Positive predictive value & $100 \%$ & $100 \%$ \\
Negative predictive value & $100 \%$ & $100 \%$ \\
\hline
\end{tabular}

conducted if it is considered as the target. Multiple values for every statistical descriptor have therefore been calculated; every value corresponds to the evaluation of one class.

\section{Results and Discussion}

Figure 2 illustrates an example of IMFs issued from EMD decomposition. It shows IMFs resulted from EMD of a signal in the dataset F (inter-ictal). The first IMFs include noise and fast waves. The last IMFs include baseline and slow waves. As indicated above, the synchronization and amplitude levels in normal EEG differ from epileptic signals. This information is inherent in IMFs temporal and spectral contents. Consequently, in the present work, all IMFs scales are taken into account in subsequent classification in order to examine the maximum temporal and spectral information level. It is noteworthy that not all of the decomposed investigated signals gave the same number of IMFs. Inter-ictal signals yielded higher number of scales than ictal and normal ones. However, all signals achieved a number within the range (8-14). Fourteen is therefore considered the maximum number taken into account. For signals yielding a number less than 14, entropy values based on empty IMFs are considered as missing values. Random Forest classifier is one of the classifiers that have the capability to deal with this case.

Table 1 illustrates a sample of entropy values of instantaneous frequencies and amplitudes for the different classes. For space reasons we have limited the values to the tenth scale. The calculated values are based on the analytic signals of IMFs after applying the DQ normalization. Absolute value of entropy decreases in slow wave IMFs.

\subsection{Healthy/ictal Recognition}

All of the normal and ictal instances have been correctly classified by the proposed method. The overall accuracy is therefore $100 \%$. Hence, the sensitivity and the specificity attain $100 \%$. Table 2 summarizes the outcomes of the normal/ictal classification.

The obtained accuracy is equal to the highest value achieved in previous literature [33] using the same EEG database as indicated in Table 3. However, EMD is 
Table 3 Results of classification (normal/ictal EEG) in literature using the same EEG database

\begin{tabular}{lll}
\hline The datasets & Method & $\begin{array}{c}\text { Achieved } \\
\text { accuracy (\%) }\end{array}$ \\
\hline Normal and ictal & Neuro-fuzzy methods applied to entropy [44] & 92.2 \\
& ANOVA applied to higher order statistics and complexity measures [45] & 92.7 \\
& Clustering applied to Hilbert transform [12] & 94 \\
& Expert model applied to discrete wavelet transform [26] & 95 \\
& Evaluated results of sample entropy (SampEn) and distribution entropy \\
& (DistEn) for EEG segments [46] & 96 \\
& PCA, KNN and SVM classification applied to statistical features [47] & 96 \\
& Artificial neural network applied to nonlinear features [48] & 97.2 \\
& Hyperbolic tangent-tangent plot [49] & 97.4 \\
& Decision tree applied to Fourier transform [32] & 98.7 \\
& Recurrent neural network applied to T-F features [2, 31] & 99.6 \\
& Artificial neural network applied to T-F Wigner-Ville features [33] \\
\hline
\end{tabular}

Table 4 Results of classification (normal/ictal/ inter-ictal EEG)

\begin{tabular}{llll}
\hline $\begin{array}{l}\text { Statistic }(\text { overall } \\
\text { accuracy }=99.7 \%)\end{array}$ & $\begin{array}{l}\text { Class: } \\
\text { normal }\end{array}$ & $\begin{array}{l}\text { Class: } \\
\text { ictal }\end{array}$ & $\begin{array}{l}\text { Class: inter-ictal } \\
\text { (epileptic zone) }\end{array}$ \\
\hline Sensitivity & $100 \%$ & $100 \%$ & $99 \%$ \\
Specificity & $100 \%$ & $99.5 \%$ & $100 \%$ \\
Positive likelihood ratio & & 200 & \\
Negative likelihood ratio & 0 & 0 & 0.01 \\
Class prevalence & $33.33 \%$ & $33.33 \%$ & $33.33 \%$ \\
Positive predictive value & $100 \%$ & $99.01 \%$ & $100 \%$ \\
Negative predictive value & $100 \%$ & $100 \%$ & $99.5 \%$ \\
\hline
\end{tabular}

nonlinear, empirical, intuitive and simpler than the exploited methods that have leaded to accuracy up to $100 \%$. WignerVille distribution used in [33] achieved $100 \%$ but was not perfectly adapted to non-linear situations. The manipulation of time windows, frequency sub-bands and resolution was therefore lengthy, imposed and not data driven. Trial-anderror based resolution and scale selection was also a disadvantage. In addition, the methods previously investigated in literature and leading to high accuracy of normal/ictal classification yield noticeably a lower accuracy when they are applied to normal/ictal/inter-ictal classification [43].

Although the works in $[10,12]$ bring into play the strong characteristics of EMD, they achieved lower accuracies than $100 \%$ due to the effect of temporal amplitude modulation of IMFs on spectral information calculation. In the present paper, the elimination of this disadvantage helped increase the accuracy to $100 \%$.

\subsection{Healthy/Ictal/Inter-ictal (F) Recognition}

According to the resulted confusion matrix, in the second classification problem, all normal and ictal instances are correctly classified. 99 out of 100 inter-ictal signals are accurately classified, as indicated in Table 4. The missed instance has been misclassified as ictal. The overall accuracy is therefore $99.7 \%$. It is higher than the accuracy values found out by other approaches utilizing the same dataset, as indicated in Table 5. This is due to the fact that Direct Quadrature method is a reliable technique that helps acquire pertinent amplitude and frequency features without overlap or redundancy. The temporal and spectral information inherent in seizure spikes is consequently represented decently by features vector.

Table 6 shows the achieved classification accuracies versus number of investigated IMFs. The outcomes demonstrate that the accuracy depends on the number of exploited IMFs. The highest accuracy is achieved when 14 IMFs are taken into account. This might explain the lower accuracies achieved in literature utilizing smaller number of IMFs [10, 12, 33]. However, close accuracy (99.3\%) is obtained when the number of selected IMFs is 5 . The outcome of normal/ictal classification varies slightly versus numbers of IMFs higher than 5. According to the results, the IMFs containing lower frequencies are also relatively informative about features distinguishing between normal, ictal and inter-ictal. In a previous work conducted by the 
Table 5 Results of classification (normal/ictal/inter-ictal EEG) in literature using the same EEG database

\begin{tabular}{|c|c|c|}
\hline The datasets & Method & $\begin{array}{l}\text { Achieved } \\
\text { accuracy }(\%)\end{array}$ \\
\hline \multirow[t]{10}{*}{ Normal, ictal and inter-ictal (seizure zone) } & Neurofuzzy network applied to discrete wavelet transform [50] & 85.9 \\
\hline & Genetic programming, K-nearest neighbour classifier [51] & 93 \\
\hline & Lyapunov exponents, artificial neural network [52] & 95 \\
\hline & Recurrent neural network applied to Lyapunov exponents [34] & 96.8 \\
\hline & Wavelet transform, K-nearest neighbour classifier [53] & 97 \\
\hline & SVM applied to fractal features [54] & 97.1 \\
\hline & Exploiting temporal correlation of EMD IMFs [55] & 98.1 \\
\hline & Naïve Bayes applied to second order difference plot (SODP) [56] & 98.7 \\
\hline & Statistical pattern recognition applied to wavelet transform [57] & 99 \\
\hline & Artificial neural network applied to time frequency features [33] & 99.2 \\
\hline \multirow{6}{*}{$\begin{array}{l}\text { Normal, ictal, inter-ictal (seizure zone) and inter- } \\
\text { ictal (opposite hemisphere) }\end{array}$} & Combined DWT and EMD applied to Morlet kernel [58] & 88.4 \\
\hline & Hyperbolic tangent—-tangent plot [49] & 92.8 \\
\hline & $\begin{array}{l}\text { Wavelet decomposition was done up to fourth level, followed by the } \\
\text { calculation of inter quartile range (IQR) [59] }\end{array}$ & 95.6 \\
\hline & Stochastic relevance analysis of short-time EEG rhythms [60] & 96.6 \\
\hline & Artificial neural network classifier with spectral features [61] & 97 \\
\hline & Artificial neural network applied to time frequency features [33] & 97.7 \\
\hline
\end{tabular}

Table 6 Result of classification (normal/ictal/inter-ictal (F) EEG) versus number of IMFs taken into account in classification

\begin{tabular}{lrrll}
\hline \multirow{2}{*}{ Number of IMFs } & \multicolumn{4}{l}{ Achieved accuracies } \\
\cline { 2 - 5 } & Healthy & Ictal & Inter-ictal (F) & Overall \\
\hline 1 & 93 & 96 & 88 & 92.3 \\
2 & 97 & 97 & 93 & 95.7 \\
3 & 99 & 97 & 97 & 97.7 \\
4 & 100 & 98 & 95 & 97.7 \\
5 & 100 & 100 & 98 & 99.3 \\
6 & 98 & 98 & 99 & 98.3 \\
7 & 100 & 97 & 99 & 98.7 \\
8 & 100 & 99 & 97 & 98.3 \\
9 & 98 & 98 & 98 & 98 \\
10 & 100 & 99 & 93 & 97.3 \\
11 & 100 & 99 & 95 & 98 \\
12 & 100 & 100 & 95 & 98.4 \\
13 & 100 & 98 & 94 & 97.4 \\
14 & 100 & 100 & 99 & 99.7 \\
\hline
\end{tabular}

first author [12], only the first four IMFs were taken into account. However, the obtained accuracy was lower (94\%).

According to the applied CFS attribute evaluator with genetic search, the features having high predictive ability are $\mathrm{f} 1, \mathrm{f} 2, \mathrm{a} 1, \mathrm{a} 2, \mathrm{a} 3, \mathrm{a} 4$ and a5. Relative contribution of every feature is illustrated in Fig. 3. The found weights of contribution of features are consistent with the findings in Table 6.

Significance of high-contribution features can also be illustrated in Fig. 4a-e that present the 2-D distribution (frequency entropy, amplitude entropy) for the scales 1, 2, 3,4 and 5, respectively. Basically, three primary clusters can be seen in every scale.

It can be illustrated from Fig. 4a-e that, in all scales from 1 to 5 , the amplitude entropy cluster centre of the IMFs resulted from ictal signals is higher than those of normal and inter-ictal. However, the difference is not obvious between the values of amplitude entropy of normal and inter-ictal clusters. These findings are consistent with Fig. 3 where the weights of a1-a5 are relatively high. On the other hand, in the high frequency scales $1-3$, centres of frequency entropy values differ between clusters of normal and inter-ictal IMFs as well as between clusters of ictal and inter-ictal IMF. These findings are consistent with Fig. 3 where the weights of $\mathrm{f} 4$ and $\mathrm{f} 5$ are small compared to $\mathrm{f} 1, \mathrm{f} 2$ and f3. The results illustrated for all clusters are also compatible with the pace of increasing of accuracies, versus number of IMFs, presented in Table 6 .

\subsection{Healthy/Ictal/Inter-ictal (F), Inter-ictal (N) Recognition}

The EEG recordings from seizure-free intervals can also be used to study the changes in the underlying dynamics of the 
cortex affected by epilepsy. The achieved accuracy of healthy/ictal/inter-ictal (F), inter-ictal $(\mathrm{N})$ classification is $98.3 \%$ as shown by Table 7. Used method is Random forest of 30 trees, each constructed while considering five random features. The obtained accuracy is higher than the accuracy values found out by other approaches utilizing the same dataset, as indicated in Table 5. According to the resulted confusion matrix, 99 normal instances have been correctly classified. One normal instance has been misclassified as inter-ictal. 97 ictal instances have been correctly classified. Three ictal instances have been misclassified as inter-ictal. 197 inter-ictal instances have been correctly classified. Two inter-ictal instances have been misclassified as normal. One inter-ictal instance has been misclassified as ictal.

The present work has many advantages. It leads to fast, low computational cost and user-friendly processing. The proposed method leads to high accuracy in comparison to other methods in literature. Furthermore, the obtained accuracy does not fall abruptly when the classification problem includes more than two classes or EEG issued from different seizure zones. The method is therefore more robust and easy-to-use than several techniques, as shown in the previous sections.

EMD application to EEG does not imply the knowledge of a priori temporal/spectral information about the signals. It is intuitively driven by the nature of the decomposed EEG time series [62]. Consequently, it does not analyze ictal, inter-ictal and normal segments by the same "Model". On the other hand, conventional time-frequency decomposition tools involve a priori assumptions that lead to pre-models insensitive to differences between epileptic signals.

EMD is not critically parameter dependent. Moreover, spectral resolution and number of achieved scales of decomposition are empirically defined, based on the inherent physiology of analyzed EEG. On the other hand, scales in Wavelet or Fourier derived transforms should be
Fig. 4 a 2-D distribution of entropy values for IMFs of scale 1 (blue normal, red ictal, green inter-ictal). $X$-axis frequency entropy. $Y$ axis amplitude entropy. b 2-D distribution of entropy values for IMFs of scale 2 (blue normal, red ictal, green inter-ictal). X-axis frequency entropy. $Y$-axis amplitude entropy. c 2-D distribution of entropy values for IMFs of scale 3 (blue normal, red ictal, green inter-ictal). $X$-axis frequency entropy. $Y$-axis amplitude entropy. d 2-D distribution of entropy values for IMFs of scale 4 (blue normal, red ictal, green inter-ictal). $X$-axis frequency entropy. $Y$-axis amplitude entropy. e 2-D distribution of entropy values for IMFs of scale 5 (blue normal, red ictal, green inter-ictal). $X$-axis frequency entropy. $Y$-axis amplitude entropy

pre-defined. Pre-definition of scales might result in missing or skipping important information/features.

In the present work, not all issued IMFs are purely mono-components. Amplitude and frequency modulation induce therefore a difficulty of instantaneous analysis. However, Direct Quadrature method overcomes the problem by the normalization system. Robust and precise calculation of features, based on accurate instantaneous values, is the main advantage of the suggested procedure.

The used random forest tree classifier is a fast tool with the advantage of open-access interpretability. Neural networks NN used in studies yielding relatively good accuracies are slower "black boxes" that do not permit easy interpretation of inherent physiological processes. Furthermore, NN imply the determination of a number of sensitive parameters values on which outcome is highly dependent.

The results of feature selection illustrate the weight of every IMF in the overall analysis as well as the significance of related temporal and spectral features. The outcome of selection yields a useful hint for further investigation of physiological processes inherent in epileptic signals, since every IMF has its own characteristics related to specific activities of neural/neuronal centres. In addition, the present work indicates the importance of the scales, higher than 4, that are not sufficiently considered or studied in literature [55].
Fig. 3 Relative contribution of every feature

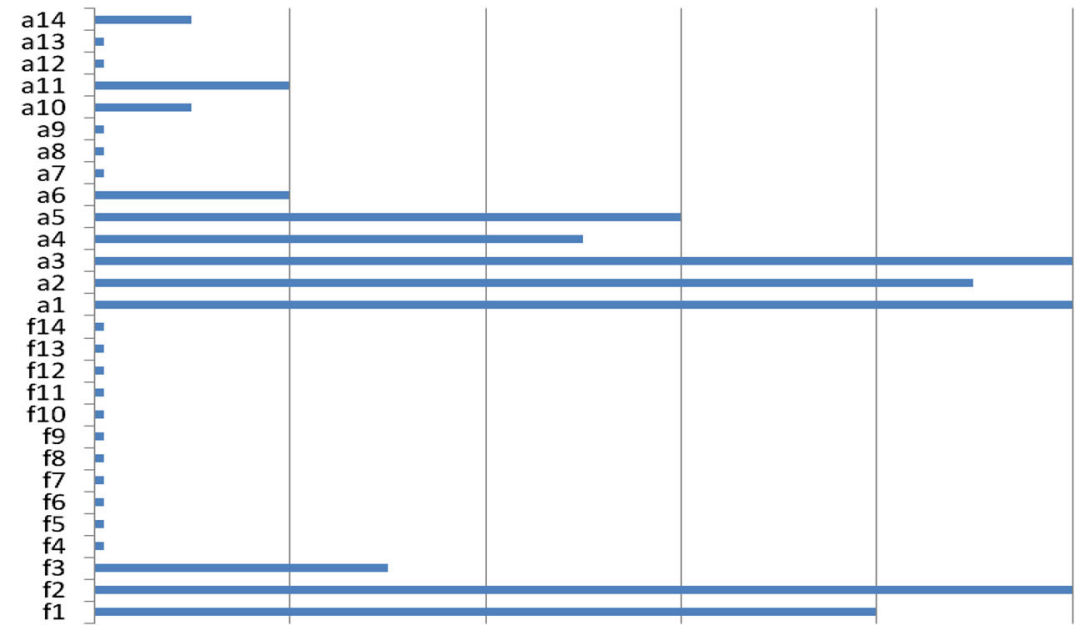


a

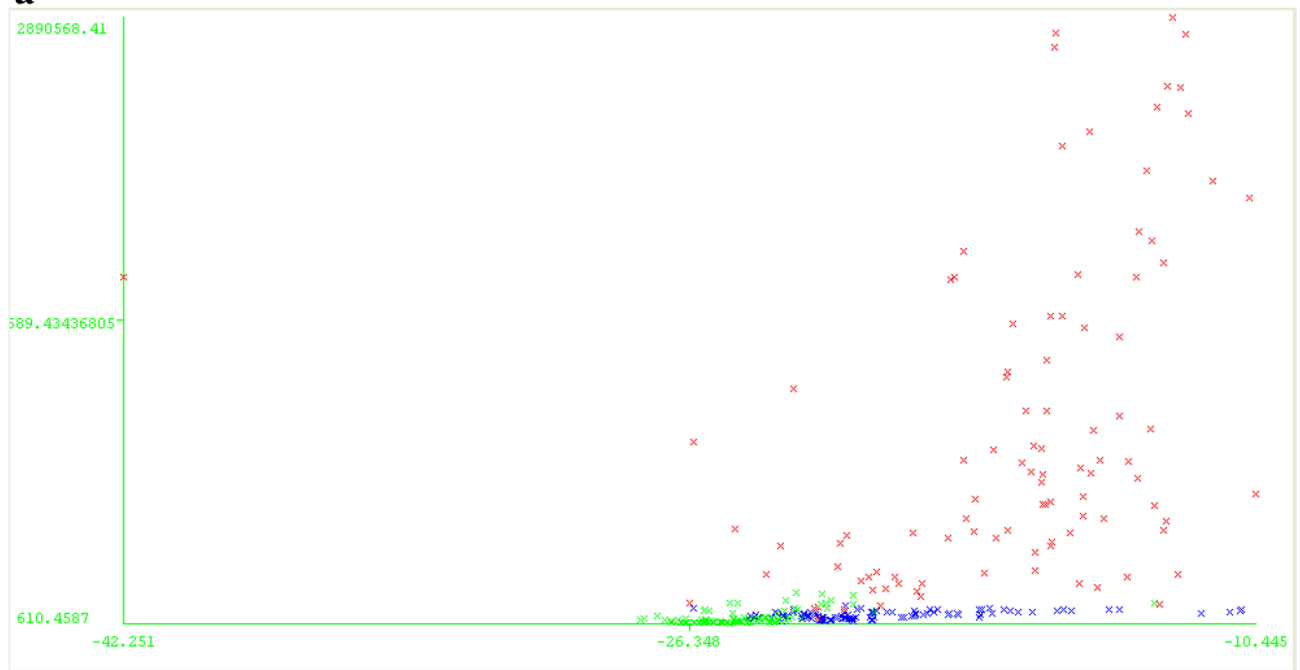

b

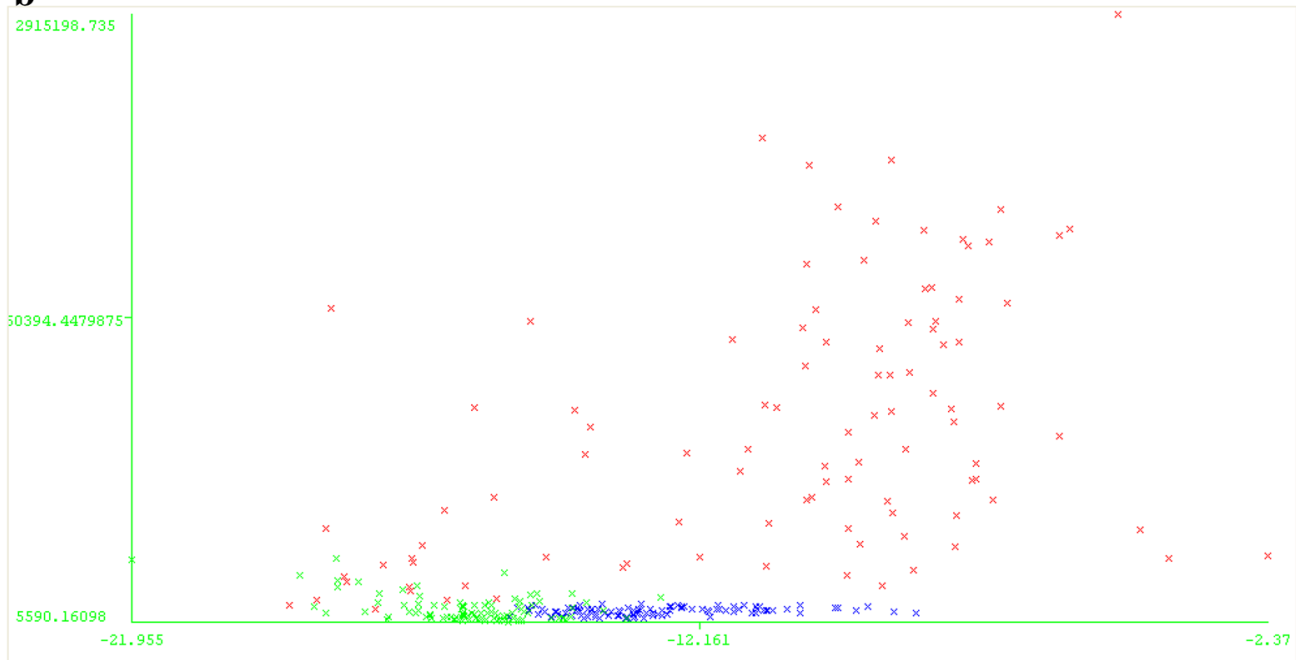

c

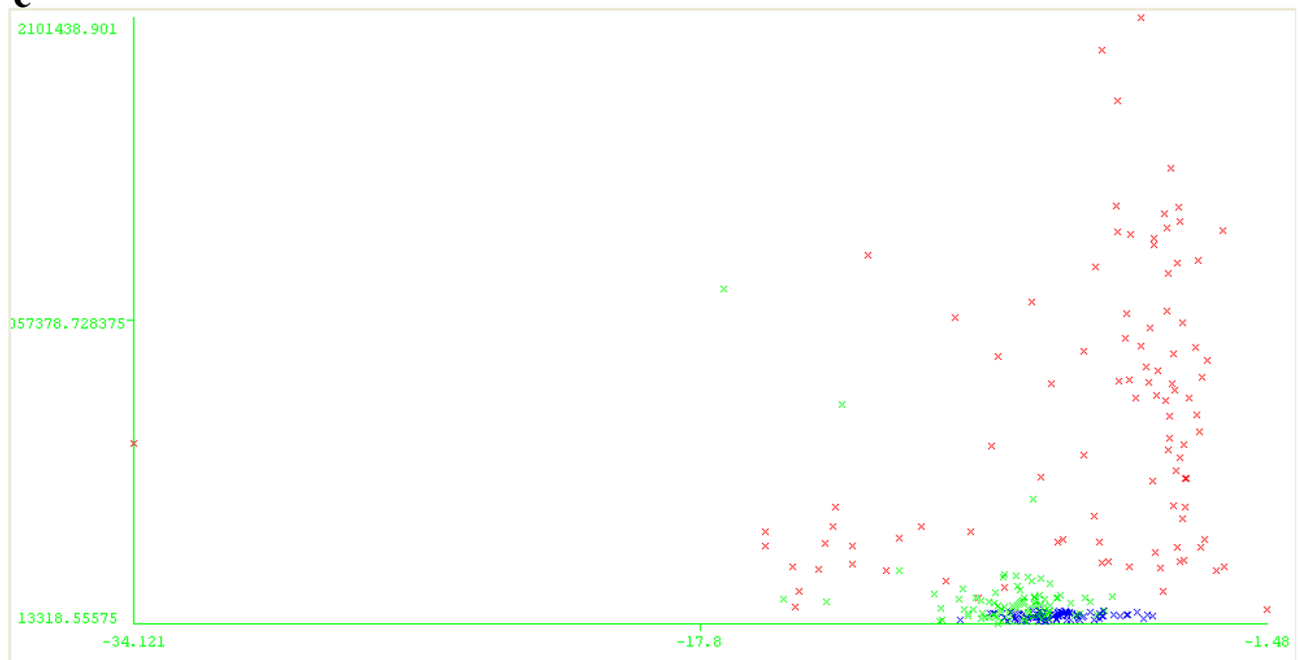



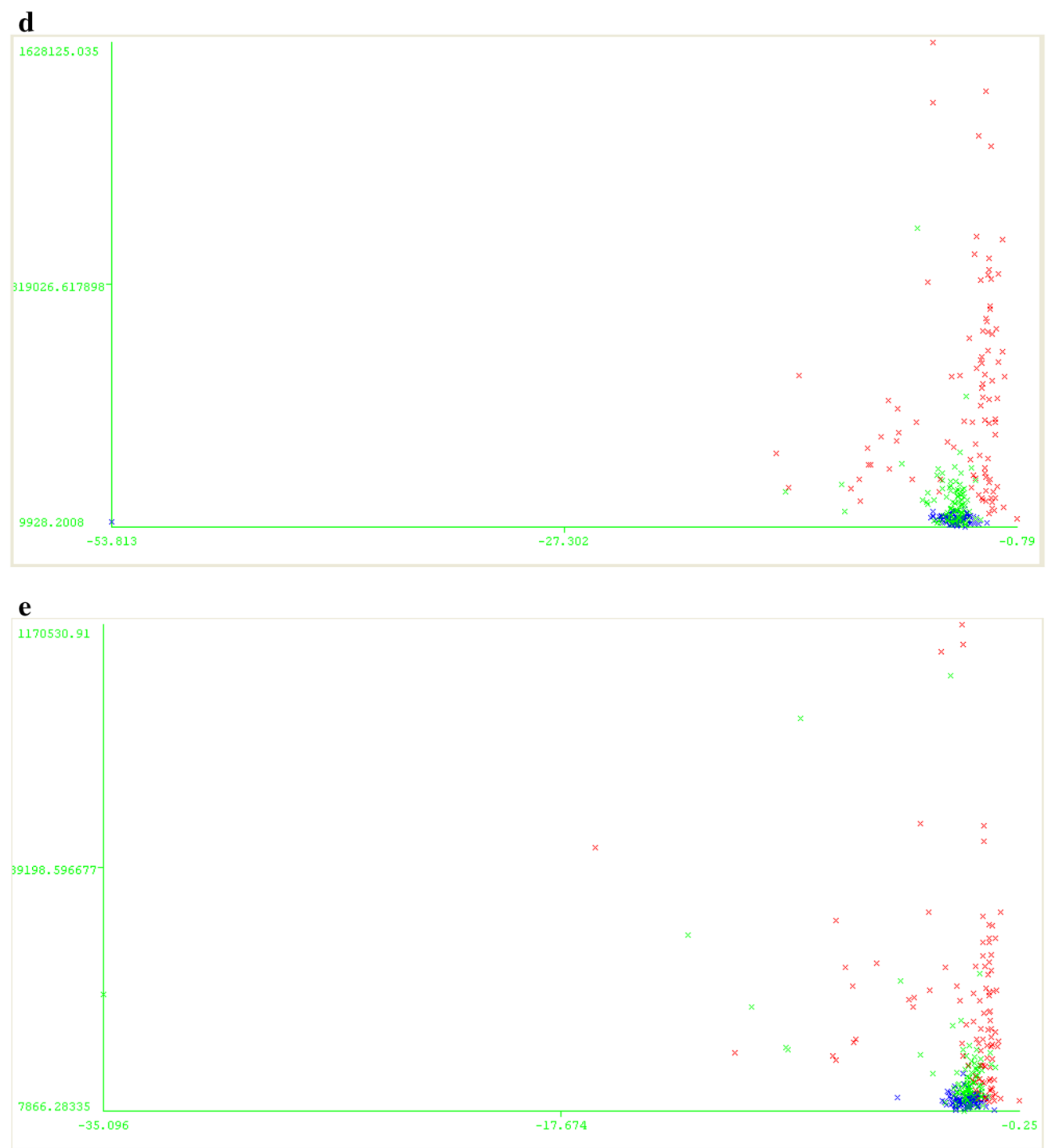

Fig. 4 continued

Table 7 Results of classification (normal/ictal/inter-ictal (F), inter-ictal (N) EEG)

\begin{tabular}{llll}
\hline Statistic (overall accuracy $=98.3 \%)$ & Class: normal & Class: ictal & Class: inter-ictal (epileptic zone + opposite hemisphere) \\
\hline Sensitivity & $99 \%$ & $97 \%$ & $98.5 \%$ \\
Specificity & $99.33 \%$ & $99.67 \%$ & $98.0 \%$ \\
Positive likelihood ratio & 148.5 & 291 & 49.25 \\
Negative likelihood ratio & 0.01 & 0.03 & 0.02 \\
Class prevalence & $25 \%$ & $25 \%$ & $50 \%$ \\
Positive predictive value & $98.02 \%$ & $98.98 \%$ & $98.01 \%$ \\
Negative predictive value & $99.67 \%$ & $99.01 \%$ & $98.49 \%$ \\
\hline
\end{tabular}

The preliminary clustering, in the present work, can lead to initial understanding of the differences, either in amplitude or frequency content (or eventually both of them), between components of normal, ictal and inter-ictal activities; this can assist practitioners in identifying the related physiological variations.

A hidden additional advantage of the proposed method is the preliminary potential of distinguishing the seizure 
zone and the opposite inter-ictal hemisphere. Results (not shown) indicate classification accuracy of normal/ictal/inter-ictal (seizure zone)/inter-ictal (opposite hemisphere) up to $85 \%$. This can partially lend a hand for detecting zones of seizure. However, more investigations should be carried out in order to improve this result and examine its applicability.

Although the proposed classification has many advantages, it has been shown that mode mixing and mode intermittency are the major limitations to the use of EMD [35]. Mode mixing indicates that oscillations of different time scales coexist in a given IMF, or that oscillations with the same time scale are assigned to different IMFs, leading to a misunderstanding of the real process. Since EMD has the disadvantage of occasional mode mixing, this might affect slightly the calculation of instantaneous values. The amplitude and frequency ratios between the components of the signal should be taken into account when mode mixing is studied, which is not always easy. Furthermore, solutions proposed in literature to avoid intermittency are either time consuming like the Ensemble Empirical Mode decomposition EEMD or unstable like the use of masking signals [37]. However, according to the obtained results, the achieved accuracy is not severely harmed by the mixing. This might be due to the fact that mixing occurs usually between consecutive IMFs that carry quasi-similar neural processes or activities from the same neural source. Since the solutions of mode mixing are time consuming or unstable, as indicated above, the performance of the suggested method should be carefully studied before application in cases of real-time seizure prediction. Mode mixing can occur in EEG segments related to transition between two different states.

The fact that a part of the calculations is based on missing values is probably considered as a disadvantage. This can be avoided by decomposing all studied signals into a fixed number of IMFs scales. This leads to counterpart IMFs in the same octave, which might make more robust clustering and classification of features. Multivariate Empirical Mode Decomposition can be one of the solutions that might be approached in future works.

\section{Conclusion}

The present work studies a robust method that helps classify EEG into normal, ictal and inter-ictal. High accuracy is achieved compared to previous literature. The intuitive characteristics of EMD, the advantages of DQ normalization, the quantification of synchronization level by entropy and the fast easy-to-interpret Random Forest classifier are the main promising elements.

In future work, application of multivariate empirical mode decomposition will be investigated in order to get the same number of IMFs for all studied EEG signals. This might help avoid features with missing values. Furthermore, additional features as variance and other types of entropy will be studied.

The suggested processing has the potential of classifying normal, ictal and inter-ictal EEG. Further directed improvements on the proposed method will allow an approach towards accurate seizures detection and management. More investigation should be conducted to study the applicability of the classification to eventual seizure prediction, especially the investigation of the effect of mode mixing. Targeted enhancements, with the help of neural mapping, might also facilitate seizure prevention prior to onset as well as guidance in neurosurgical interventions.

\section{References}

1. Sharma, R., Pachori, R. B., \& Acharya, U. R. (2015). Application of entropy measures on intrinsic mode functions for the automated identification of focal electroencephalogram signals. Entropy, 17, 669-691.

2. Srinivasan, V., Eswaran, C., \& Sriraam, N. (2005). Artificial neural network based epileptic detection using time-domain and frequency domain features. Journal of Medical Systems, 29(6), 647-660.

3. Tzallas, A. T., Karvelis, P. S., Katsis, C. D., Fotiadis, D. I., Giannopoulos, S., \& Konitsiotis, S. (2006). A method for classification of transient events in EEG recordings: Application to epilepsy diagnosis. Methods of Information inMedicine, 45(6), 610-621.

4. Mormann, F., Lehnertz, K., David, P., \& Elger, C. E. (2000). Mean phase coherence as a measure for phase synchronization and its application to the EEG of epilepsy patients. Physica D: Nonlinear Phenomena, 144, 358-369.

5. Lehnertz, K., \& Elger, C. E. (1995). Spatio-temporal dynamics of the primary epileptogenic area in temporal lobe epilepsy characterized by neuronal complexity loss. Electroencephalography and Clinical Neurophysiology, 95, 108-117.

6. Prior, P. F., Virden, R. S. M., \& Maynard, D. E. (1973). An EEG device for monitoring seizure discharges. Epilepsia, 14(4), 367-372.

7. Gotman, J. (1982). Automatic recognition of epileptic seizures in the EEG. Electroencephalography and Clinical Neurophysiology, 54(5), 530-540.

8. Webber, W. R. S., Lesser, R. P., Richardson, R. T., \& Wilson, K. (1996). An approach to seizure detection using an artificial neural network (ANN). Electroencephalography and Clinical Neurophysiology, 98(4), 250-272.

9. Harding, G. W. (1993). An automated seizure monitoring system for patients with indwelling recording electrodes. Electroencephalography and Clinical Neurophysiology, 86(6), 428-437.

10. Pachori, R. B. (2008). Discrimination between ictal and seizurefree EEG signals using empirical mode decomposition. The IEEE Signal Processing Letters. doi:10.1155/2008/293056.

11. Huang, N., Shen, Z., Long, S., Wu, M., Shih, H. H., Zheng, Q., et al. (1998). The empirical mode decomposition and the Hilbert spectrum for nonlinear and non-stationary time series analysis. Proceedings of the Royal Society A: Mathematical, Physical and Engineering Sciences, 454, 903-995. 
12. Oweis, R., \& Abdulhay, E. (2011). Seizure identification in EEG signals utilizing Huang and Hilbert transforms. BioMedical Engineering OnLine, 10, 38.

13. Pachori, R. B., \& Bajaj, V. (2011). Analysis of normal and epileptic seizure EEG signals using empirical mode decomposition. Computer Methods and Programs in Biomedicine, 104, 373-381.

14. Pachori, R. B., Sharma, R., \& Patidar, S. (2015). Classification of normal and epileptic seizure EEG signals based on empirical mode decomposition. Complex System Modelling and Control through Intelligent Soft Computations, 319, 367-388.

15. Pachori, R. B., \& Patidar, S. (2014). Epileptic seizure classification in EEG signals using second-order difference plot of intrinsic mode functions. Computer Methods and Programs in Biomedicine, 113, 494-502.

16. Sharma, R., \& Pachori, R. B. (2015). Classification of epileptic seizures in EEG signals based on phase space representation of intrinsic mode functions. Expert Systems with Applications, 42, $1106-1117$.

17. Kumar, T. S., Kanhangad, V., \& Pachori, R. B. (2014). Classification of seizure and seizure-free EEG signals using multi-level local patterns. In Proceedings of the IEEE 19th international conference on digital signal processing, Hong Kong (pp. 646-650).

18. Li, S., Zhou, W., Yuan, Q., Geng, S., \& Cai, D. (2013). Feature extraction and recognition of ictal EEG using EMD and SVM. Computers in Biology and Medicine, 43, 807-816.

19. Zhu, G., Li, Y., Wen, P. P., Wang, S., \& Xi, M. (2013). Epileptogenic focus detection in intracranial EEG based on delay permutation entropy. AIP Conference Proceedings, 1559, 31-36.

20. Sharma R, Pachori, R. B., \& Gautam, S. (2014). Empirical mode decomposition based classification of focal and non-focal EEG signals, In Proceedings of the international conference on medical biometrics, Shenzhen (pp. 135-140).

21. Orosco, L., Correa, A. G., \& Laciar, E. (2010). Multiparametric detection of epileptic seizures using empirical mode decomposition of eeg records. In Proceedings of 32nd annual international conference of the IEEE EMBS Buenos Aires (pp. 951-954).

22. Kiranmayi, G. R., \& Udayashankara, V. (2014). EEG subband analysis using approximate entropy for the detection of epilepsy. IOSR Journal of Computer Engineering, 16(5), 21-27.

23. Adeli, H., Dastidar, S. G., \& Dadmehr, N. (2007). A waveletchaos methodology for analysis of EEGs and EEG subbands to detect seizure and epilepsy. IEEE Transactions on Biomedical Engineering, 54(2), 205-211.

24. Dastidar, S. G., Adeli, H., \& Dadmehr, N. (2007). Mixed-band wavelet-chaos-neural network methodology for epilepsy and epileptic seizure detection. IEEE Transactions on Biomedical Engineering, 54(9), 1545-1551.

25. Dastidar, S. G., Adeli, H., \& Dadmehr, N. (2008). Principal component analysis-enhanced cosine radial basis function neural network for robust epilepsy and seizure detection. IEEE Transactions on Biomedical Engineering, 55(2), 512-518.

26. Subasi, A. (2007). EEG signal classification using wavelet feature extraction and a mixture of expert model. Expert Systems with Applications, 32(4), 1084-1093.

27. Wang, C. M., Zou, J.-Z., Zhang, J., Zhang, Z.-S., \& Zhang, C.-M. (2009). Classifying detection of epileptic EEG based on approximate entropy in wavelet domain. In Proceedings of the IEEE conference on bio medical engineering and informatics (pp. $1-5)$.

28. Guo, L., Rivero, D., \& Pazos, A. (2010). Epileptic seizure detection using multiwavelet transform based approximate entropy and artificial neural networks. Journal of Neuroscience Methods, 193, 156-163.
29. Vavadi, H., Ayatollahi, A., \& Mirzaei, A. (2010). A waveletapproximate entropy method for epileptic activity detection from EEG and its sub-bands. Journal Biomedical Science and Engineering, 3, 1182-1189.

30. Tzallas, A. T., Tsipouras, M. G., \& Fotiadis, D. I. (2009). Epileptic seizure detection in EEGs using time-frequency analysis. IEEE Transactions on Information Technology in Biomedicine, 13(5), 703-710.

31. Srinivasan, V., Eswaran, C., \& Sriraam, N. (2007). Approximate entropy-based epileptic EEG detection using artificial neural networks. IEEE Transactions on Information Technology in Bio Medicine, 11(3), 288-295.

32. Polat, K., \& Günes, S. (2007). Classification of epileptiform EEG using a hybrid system based on decision tree classifier and fast Fourier transform. Applied Mathematics and Computation, 32(2), $625-631$.

33. Tzallas, T., Tsipouras, M. G., \& Fotiadis, D. I. (2007). Automatic seizure detection based on time-frequency analysis and artificial neural networks. Computational Intelligence and Neuroscience, 7(3), 1-13.

34. Güler, N. F., Ubeyli, E. D., \& Güler, I. (2005). Recurrent neural networks employing Lyapunov exponents for EEG signals classification. Expert Systems with Applications, 29(3), 506-514.

35. Abdulhay E, Guméry PY, Fontecave-Jallon J, Baconnier P. (2009). Cardiogenic oscillations extraction in inductive plethysmography: Ensemble empirical mode decomposition. In IEEE EMBS proceedings, Minnesota (pp. 2240-2243).

36. Andrzejak, R. G., Lehnertz, K., Mormann, F., Rieke, C., David, P., \& Elger, C. E. (2001). Indications of nonlinear deterministic and finite-dimensional structures in time series of brain electrical activity: Dependence on recording region and brain state. Physical Review E: Statistical, Nonlinear, and Soft Matter Physics, 64, 061907-1-061907-8.

37. Huang, N. E., \& Wu, Z. (2008). A review on Hilbert-Huang transform: Method and its applications to geophysical studies. Reviews of Geophysics, 46, 228-251.

38. Kschischang, F. R. (2006). The Hilbert Transform. Toronto: University of Toronto.

39. https://en.wikipedia.org/wiki/Hilbert_transform, visited in June 2017.

40. Random, F. T., \& Leo, B. (2001). Random forests. Machine Learning., 45(1), 5-32.

41. https://en.wikipedia.org/wiki/Cross-validation_(statistics), visited in May 2016.

42. Seni, G., \& Elder, J. F. (2010). Ensemble methods in data mining: Improving accuracy through combining predictions. Synthesis Lectures on Data Mining and Knowledge Discovery, 2(1), 1-126.

43. Das, A. B., Bhuiyan, M. I. H., \& Alam, S. M. S. (2016). Classification of EEG signals using normal inverse Gaussian parameters in the dual-tree complex wavelet transform domain for seizure detection. Signal Image and Video Processing, 10(2), 259-266.

44. Kannathal, N., Choo, M. L., Acharya, U. R., \& Sadasivan, P. K. (2005). Entropies for detection of epilepsy in EEG. Computer Methods and Programs in Biomedicine, 80(3), 187-194.

45. Palani Thanaraj, K., \& Chitra, K. (2014). Multichannel feature extraction and classification of epileptic states using higher order statistics and complexity measures. International Journal of Engineering and Technology, 6(1), 102-109.

46. Li, P., Karmakar, C., Yan, C., Palaniswami, M., \& Liu, C. (2016). Classification of 5-S epileptic EEG recordings using distribution entropy and sample entropy. Frontiers in Physiology, 7, 136.

47. Noertjahjani, S., Susanto, A., Hidayat, R., \& Wibowo, S. (2016). Ictal epilepsy and normal eeg feature extraction based on PCA, KNN and SVM classification. Journal of Theoretical and Applied Information Technology, 83(1), 100-106. 
48. Nigam, V. P., \& Graupe, D. (2004). A neural-network-based detection of epilepsy. Neurological Research, 26(1), 55-60.

49. Karimoi, R. Y., \& Karimoi, A. Y. (2014). Classification of EEG signals using hyperbolic tangent-tangent plot. International Journal of Intelligent Systems and Applications, 08, 39-45.

50. Sadati, N., Mohseni, H. R., \& Maghsoudi, A. (2006). Epileptic seizure detection using neural fuzzy networks. In Proceedings of IEEE international conference on fuzzy systems, Vancouver (pp. 596-600).

51. Guo, L., Rivero, D., Dorado, J., Munteanu, C. R., \& Pazos, A. (1042). Automatic feature extraction using genetic programming: An application to epileptic EEG classification. Expert Systems with Applications, 2011, 38.

52. Ubeyli, E. D. (2006). Analysis of EEG signals using Lyapunov exponents. Neural Network World, 16(3), 257.

53. Orhan, U., Hekim, M., \& Ozer, M. (2011). EEG signals classification using the K-means clustering and a multilayer perceptron neural network model. Expert Systems with Applications, 38, 13475.

54. Wang, Y., Zhou, W., Yuan, Q., Li, X., Meng, Q., Zhao, X., et al. (2013). Comparison of ictal and interictal EEG signals using fractal features. International Journal of Neural Systems, 23(6), 1350028.

55. Parvez, M. Z., Paul, M., \& Antolovich, M. (2015). Detection of pre-stage of epileptic seizure by exploiting temporal correlation of EMD decomposed EEG signals. Journal of Medical and Bioengineering, 4(2), 110-116.

56. Yayik, A., Yildirim, E., Kutlu, Y., \& Yildirim, S. (2014). Epileptic state detection: Pre-ictal, inter-ictal, ictal. International
Journal of Intelligent Systems and Applications in Engineering, 3(1), 14-18.

57. Gajic, D., Djurovic, Z., Di Gennaro, S., \& Gustafsson, F. (2014). Classification of EEG signals for detection of epileptic seizures based on wavelets and statistical pattern recognition. Biomedical Engineering: Applications, Basis and Communications, 26(2), 1450021.

58. Parvez, M. Z., \& Paul, M. (2014). Epileptic seizure detection by analyzing EEG signals using different transformation techniques. Neurocomputing, 145, 190-200.

59. Thasneem, F., Bedeeuzzaman, M., \& Paul, J. (2013). Wavelet based features for classification of normal, ictal and interictal EEG signals. Journal of Medical Imaging and Health Informatics, 3(2), 301-305.

60. Duque-Muñoz, L., Espinosa-Oviedo, J. J., \& Castellanos-Dominguez, C. G. (2014). Identification and monitoring of brain activity based on stochastic relevance analysis of short-time EEG rhythms. BioMedical Engineering OnLine, 13, 123.

61. Ramgopal, S., Thome-Souza, S., Jackson, M., Kadish, N. E., Fernández, I. S., Klehm, J., et al. (2014). Seizure detection, seizure prediction, and closed-loop warning systems in epilepsy. Epilepsy \& Behavior, 37, 291-307.

62. Argoud, F. I. M., de Azevedo, F. M., Neto, J. M., \& Grillo, E. (2006). SADE3: An effective system for automated detection of epileptiform events in long-term EEG based on context information. Medical \& Biological Engineering \& Computing, 44(6), 459-470. 